\title{
Gesunder Schlaf ist der Schlüssel
}

\section{Innovative Matratzentechnologie steigert Wirtschaftlichkeit in Heimen}

" Vier von zehn Menschen über 65 Jahren leiden in Deutschland unter massiven Schlafstörungen. Studien zeigen, dass etwa 70\% der Bewohner in Pflegeheimen heute an Demenz leiden. Hinzu kommt, dass Schlafmangel den Verlauf vieler Erkrankungen verschlimmert. Senioren, die gut durchschlafen, sind gesünder und zufriedener. Sie sind in der Lage, mit mehr Energie am Tagesgeschehen teilzunehmen, lassen sich besser aktivieren und verfügen über höhere Lebensqualität. Auch für die Mitarbeiter bedeutet dies eine Entlastung. Die besseren Arbeitsbedingungen führen $\mathrm{zu}$ weniger Krankschreibungen und Fluktuation. So wirkt sich der gesunde Schlaf der Bewohner langfristig auch auf die Wirtschaftlichkeit der Einrichtung aus.

„Schlafstörungen und der somit erhöhte Betreuungsaufwand ließen sich mit einer Demenzmatratze durchaus vermeiden", erklärt Marion Saller vom Institut für Innovationen im Gesundheitswesen und angewandte Pflegeforschung. Die Pflegeexpertin bezieht sich dabei auf bewährte Therapiematratzen mit sogenannter MiS Micro-Stimulation. Die Systeme bestehen aus zwei Komponenten: einer Unterfederung mit flexibler Liegefläche aus Flügelfedern sowie einer passenden Matratze aus atmungsaktivem, antibakteriellem Polyurethanschaumstoff. Die MiS-Systeme verwandeln Mikrobewegungen, sogar Atembewegungen, automatisch in mikrofeine Gegenbewegungen. Dadurch gewinnen Demenzpatienten an Orientierung. Sie fühlen sich geborgen und kommen schneller wieder zur Ruhe, wenn sie nachts doch einmal wach werden.

Auch Patienten mit Parkinson, chronischen Schmerzen oder Dekubitus profitieren von der Technologie. Versteifungen und Schmerzen werden durch die Spezialmatratzen vermieden. Bestehende Wundgeschwüre heilen aus. Zusätzlich zeichnen sich die Matratzen dadurch aus, dass sie lang- lebig, einfach zu reinigen, wiedereinsatzfähig und wartungsfrei sind. Durch verschiedene Optionen zur Finanzierung, wie Leasing oder Miete, sind die neuen Spezialmatratzen auch für Einrichtungen interessant, die wirtschaftlich handeln müssen und Ressourcen sparen wollen.

\section{www.thomashilfen.de}

\section{Supportive Onkologie}

\section{Gegen Mukositis}

" In der Pipeline von Mundipharma im Bereich Supportive Care in der Onkologie befindet sich eine gebrauchsfertige Mundspüllösung zur Behandlung der oralen Mukositis bei Tumortherapie (Ectomusal ${ }^{\circledR}$ ). Das Produkt enthält Ectoin ${ }^{\oplus}$, das ein Bestandteil extremophiler Bakterien ist und es diesen ermöglicht, in extremem Milieu zu überleben. Ectoin hat durch die Ausbildung stabiler Wasserstoffbrückenbindungen einen hydratisierenden Effekt. Die Stabilisierung von Zellmembranen stärkt die Barrierefunktion und reduziert Entzündungen.

Pressekonferenz „Ectomusal ${ }^{\circledR}$ - Mundspüllösung gegen orale Mukositis bei Tumorpatienten" beim DGHO-Kongress 2017; Stuttgart, 30.09.2017 (Veranstalter: Mundipharma Deutschland GmbH \& Co.KG)

\section{Schwindel ist meist multikausal}

\section{Risikoindikator für Stürze, Synkopen, Pflegebedürftigkeit}

" Schwindel ist eines der häufigsten Symptome bei älteren Menschen. Die Anamnese bei älteren Patienten sollte daher Art, Auslöser, Begleitsymptome und die Frage nach Bewusstseinsverlust ebenso einschließen wie die genaue Medikation inklusive der OTCMedikamente. Indiziert ist auch die körperliche neurologische Untersuchung.

Therapeutische Ansatzpunkte sind zunächst die Suche und Behandlung eventuell vorhandener monokausaler Schwindelursachen, so der Geriater Prof. Karl-Günter Gaßmann. Indiziert und oft sehr erfolgreich bei gemischtem und multifaktoriellem Schwindel seien das Training von Gangsicherheit, Gleichgewicht, Kraft sowie ein Verhaltenstraining, das sich auch der Bewältigung der Angst vor Stürzen widme. Nimmt der Patient schwindelinduzie- rende Medikamente, sollte eine Umstellung oder ein Auslassversuch mit nachfolgendem Ausschleichen probiert werden. Die medikamentöse Schwindeltherapie greife bei gewisser Indikation, sollte aber keine Dauertherapie sein. Als einziges natürliches Präparat wird Vertigoheel ${ }^{\circledR}$ bei akutem Schwindel seit einem Jahr in der hausärztlichen DEGAM-Leitlinie empfohlen. Wegen des Fehlens von Nebenund Wechselwirkungen eignet es sich vor allem für multimorbide ältere $\mathrm{Pa}$ tienten und wird von vielen GKV als freiwillige Satzungsleistung erstattet.

(hae)

Lunchsymposium „Schwindel - eine interdisziplinäre Herausforderung “ anlässlich der Jahrestagung der DGG; Frankfurt/M., 29.09.2017

(Veranstalter: Heel GmbH) 